\title{
Electronic Transport of Uranium Mononitride
}

\author{
Barbara Szpunar ${ }^{1 *}$, Jayangani I. Ranasinghe1, Jerzy A. Szpunar ${ }^{2}$ \\ ${ }^{1}$ Department of Physics and Engineering Physics, University of Saskatchewan, Saskatoon, Canada \\ ${ }^{2}$ Department of Mechanical Engineering, University of Saskatchewan, Saskatoon, Canada \\ Email: *B.Szpunar@usask.ca
}

How to cite this paper: Szpunar, B., Ranasinghe, J.I. and Szpunar, J.A. (2021) Electronic Transport of Uranium Mononitride. Journal of Modern Physics, 12, 1409-1417. https://doi.org/10.4236/jmp.2021.1210084

Received: April 14, 2021

Accepted: August 1, 2021

Published: August 4, 2021

Copyright $\odot 2021$ by author(s) and Scientific Research Publishing Inc. This work is licensed under the Creative Commons Attribution International License (CC BY 4.0).

http://creativecommons.org/licenses/by/4.0/ (c) (i) Open Access

\begin{abstract}
We investigated the electronic heat capacity, thermal conductivity, and resistivity of UN using Quantum Espresso and EPW code. GGA, PBEsol functional was used. The calculated electronic heat coefficient was found to be significantly reduced $\left(0.0176 \mathrm{~J} \cdot \mathrm{mol}^{-1} \cdot \mathrm{K}^{-2}\right.$ versus $\left.0.0006 \mathrm{~J} \cdot \mathrm{mol}^{-1} \cdot \mathrm{K}^{-2}\right)$ when the non-local hybrid functional (B3LYP) was used. Furthermore, we calculated electrical resistivity using a very transparent Ziman's formula for metals with the Eliashberg transport coupling function as implemented in EPW code for non-spin-polarized calculations. The number of mobile electrons in UN, as a function of temperature, was derived from the ratio of the calculated resistivity and available experimental data. The electronic thermal conductivity was evaluated from the calculated electronic resistivity via Wiedemann-Franz law with the number of mobility electrons $\left(n_{a v}\right)$ incorporated (averaged over the temperature range $300 \mathrm{~K}-1000 \mathrm{~K}$ ). Both the electronic thermal conductivity and resistivity, as calculated using newly evaluated $n_{a n}$ compare well with experimental data at $\sim 700 \mathrm{~K}$, but to reproduce the observed trend as a function of temperature, the number of mobile electrons must decrease with the temperature as evaluated.
\end{abstract}

\section{Keywords}

UN, Electronic Thermal Conductivity, Electronic Structure, Number of Mobility Electrons, Quantum ESPRESSO, EPW Codes

\section{Introduction}

Urania fuel, which is used in conventional nuclear reactors, is not suitable for some designs of new generation reactors (e.g., SuperCritical Water Reactor) due to its low thermal conductivity [1]. In the context of finding a sustainable development solution to the use of non-renewable energy sources, innovative research towards enhanced accident-tolerant nuclear fuel (EATF) that can with- 
stand the loss of coolant for a long time is gaining momentum. EATF materials must have higher thermal conductivities to prevent meltdown [2]. High-density metallic compounds, uranium silicide $\left(\mathrm{U}_{3} \mathrm{Si}_{2}\right)$ and uranium and thorium nitrides (UN, ThN) [3], have been proposed as alternative EATFs [2] for implementation as lower enrichment fuel.

In our previous papers [4] [5], we have investigated UN, which has the same cubic structure ( $F m \overline{3} m$ symmetry) as ThN, and may be used in combination to enhance thermal conductivity as both are metals. In these metallic fuels, thermal conductivity does not deteriorate with increasing temperature like the lattice-governed thermal conductivity in insulators (e.g. urania [6]). This is due to the increasing presence of electronic carriers with mobility as temperature rises. Since both electronic conductivity and electronic contribution to thermal conductivity are related to electron mobility, they can be derived from each other via the Wiedemann-Franz proportionality law (WFL), which is very useful in determining the contribution from electrons to the measured total thermal conductivity.

Enhanced computational capabilities have led to significant developments in extending the potentialities of based on density functional theory (DFT) codes. $A b$ initio calculations based on DFT have become an essential theoretical tool in investigating novel nuclear materials. In this study, we used first-principles, predictive calculations based on DFT, where ground state energy is calculated using functionals dependent on the electronic density only. Unlike urania, fewer such studies have been done on these alternative fuels. In particular number of mobility, electrons need to be investigated, since they are crucial in enhancing the thermal conductivity of metals at high temperatures. High thermal conductivity in metallic fuels allows for fast heat dissipation and makes reactors safer and more economical.

In an evaluation of the electronic heat capacity, very accurate calculations of electrondensities of states are required. In our previous work on thoria [7], we found that the non-local hybrid functional (B3LYP) [8] modified the electronic structure significantly and led to a larger bandgap. It was therefore of interest to examine the electronic structure of UN using B3LYP to find how it might affect the value of the electronic heat capacity coefficient of $U N$, as evaluated here.

\section{Calculation Methodology}

To evaluate the geometrical and electronic structures of UN we used Quantum ESPRESSO (QE) code [9], since there is already an interface provided between QE and EPW (Electron-Phonon coupling using Wannier functions) code [10], which we used to evaluate electronic transport.

We calculated the electronic heat capacity coefficient $(\gamma)$, which is proportional to the electron density of states at Fermi energy at the equilibrium lattice constants using the density of states of electrons per eV at the Fermi energy $\left(\rho\left(\varepsilon_{F}\right)\right)$ for UN from: 


$$
C_{e}(T)=\pi / 3 \times 6.242 \times 10^{18} \rho\left(\varepsilon_{F}\right) N_{A} k_{B}^{2} T \equiv \gamma T
$$

The respective electronic heat capacity is proportional to $\gamma$ and increases linearly with temperature and can be evaluated using Equation (1). A very accurate evaluation of the electronic structure was required; therefore, in addition to generalized gradient approximation (GGA) of the Perdew, Burke, and Ernzerhof functional developed for solids (PBEsol) [11] DFT, we used non-local Becke three-parameter hybrid exchange (B3)+LYP functional (B3LYP) [8] to modify the electronic structure around Fermi energy, and the respective electronic heat coefficients were compared. We used the same norm-conserved pseudopotentials and setup for the electronic structure calculations as detailed in our previous work on UN [5].

Using the density functional perturbation theory (DFPT) method as implemented in QE code [12], we evaluated previously [5] the phonons' dispersion relation and the densities of states of UN.

We computed the electrical resistivity calculation $\left(\rho_{\text {calc }}(T)\right)$ using a very transparent Ziman's formula for metals (Equation 54 in Ref. [10]) with the Eliashberg transport coupling function: $\alpha_{t r}^{2} F(\omega)$ (Equation 55 in Ref. [10]) and a hardcoded number of mobility electrons (nc) per cell equal to 8 (assumed for lead as an example) as implemented in EPW code [10]:

$$
\rho_{\text {calc }}(T)=\frac{4 \pi m_{e}}{n e^{2} k_{B} T} \times \int_{0}^{\infty} \mathrm{d} \omega \hbar \alpha_{t r}^{2} F(\omega) n(\omega, T)[1+n(\omega, T)]
$$

where $n=$ nc/omega and omega is the calculated in the code volume of the primitive cell in a.u.. Note that in the new version (QE 6.7) nc is a parameter with the default value equal to (4).

We also calculated integrated electron-phonon strength $(\lambda)$ as a function of frequency $(\omega)[10]$ :

$$
\lambda=\int_{0}^{\omega} \frac{\alpha_{t r}^{2} F(\omega)}{\omega} \mathrm{d} \omega
$$

The cumulative electron-phonon strength and Eliashberg transport coupling function could be used in future comparisons with other metallic fuels. Additionally, since the experimental resistivity is known for UN, we evaluated the effective number of electron carriers in electronic transport $\left(n_{\text {eff }}(T)\right)$ :

$$
n_{\text {eff }}(T)=\frac{8 \rho(T)_{\text {calc }}}{\rho(T)_{\text {exp }}}
$$

When replacing $\mathrm{n}$, the number of mobility electrons in Equation (2) with $n_{\text {eff }}(T)$, the calculated resistivity would become equal to the experimental. We also calculated averages of an effective number of electron carriers in the $300 \mathrm{~K}$ $1000 \mathrm{~K}$ temperature range $\left(n_{a v}\right)$ and calculated resistivity by replacing $\mathrm{n}$ with $n_{a v}$

The electronic contribution to the thermal conductivity $\left(\kappa_{e}\right)$ can be calculated via Wiedemann-Franz law [13] from the electrical conductivity $(\sigma)$ or resistivity $\left(\rho(T)=\sigma(T)^{-1}\right)$ 


$$
\kappa_{e}=\frac{\pi}{3}\left(\frac{k_{B}}{e}\right)^{2} \sigma T
$$

where $k_{B}$ is the Boltzmann constant, $e$ is the electron charge, and $T$ is the temperature in $\mathrm{K}$. We calculated electronic thermal conductivity using the $n_{a v}$ number of mobility electrons of Un and compared results with the experiment.

\section{Results and Discussion}

Here we adopted for UN the same parameters and norm-conserving pseudopotentials: U.pbesol-n-nc.UPF and N.pbesol-nc.UPF from QE code as in our previous studies [5]. We assumed $5 \mathrm{f}^{3}, 6 \mathrm{~d}^{1}, 7 \mathrm{~s}^{2}$ for $\mathrm{U}$ and $2 \mathrm{~s}^{2}, 2 \mathrm{p}^{3}$ for $\mathrm{N}$ as the electronic configurations. The evaluated lattice constants for non-magnetic UN $(0.489 \mathrm{~nm})$ and using this setup [5] agreed very well with the experimental value of 0.489 [14].

\subsection{Electrons' Density of States and Electronic Heat Capacity}

Using QE code, we previously performed [5] non-spin-polarized calculations to evaluate the electronic structure of UN at the equilibrium lattice constants. In addition, we used here the non-local hybrid exchange functional (B3LYP) [8] to try to modify its electronic structure around Fermi energy as demonstrated before during an evaluation of the bandgap of $\mathrm{ThO}_{2}$ [7]. However, there are no pseudopotentials developed for B3LYP either for $\mathrm{N}$ or $\mathrm{U}$ atoms and the calculations are very computationally demanding. Therefore, we reduced the kinetic energy cutoff for wave functions to $200 \mathrm{Ry}(2721 \mathrm{eV})$ and used existing pseudopotentials for other functionals: N.blyp-hgh.UPF and the used above U.pbesoln-nc.UPF. Otherwise, we used a similar setup and the same lattice constants as previously determined for PBEsol calculations [5].

In Figure 1(a) and Figure 1(b), we compare the electronic structure of UN evaluated using a) PBEsol and b) B3LYP functionals. We present the evaluated per formula unit (f.u.,: UN) total electronic density of states of UN (solid black line) together with the projected partial electron densities of states (plotted with $0.1 \mathrm{eV}$ energy step) of nitrogen: $2 \mathrm{p}$ (dashed-dot-dot blue line), and $2 \mathrm{~s}$ (dashedlong pink line) and for uranium: $6 \mathrm{~d}$ (dark red dotted line) and $5 \mathrm{f}$ (dashed medium green line) electron densities of states. The Fermi energy is indicated by the grey dashed line. The integrated (with $0.01 \mathrm{eV}$ energy step) total number of electrons as a function of energy is indicated as dashed-dot red lines, with the total number of electrons at Fermi energy: 11e. In both calculations (Figure 1(a) and Figure 1(b)) $2 \mathrm{p}$ electrons of the $\mathrm{N}$ atom are located below $10 \mathrm{eV}$ while $2 \mathrm{~s}$ electrons are located below $0 \mathrm{eV}$ energy.

Similar to our previous results for thoria [7], we found that the non-local hybrid functional B3LYP pushed $5 f$ electrons of $U$ up and away from Fermi energy (Figure 1(b)) when compared with our calculations using PBEsol in Figure 1(a). This resulted in a lower density of states at Fermi energy $\left(\rho\left(\varepsilon_{F}\right)\right)$ as the hybridized states of $6 \mathrm{~d}$ and $5 \mathrm{f} \mathrm{U}$ electrons also moved up just above it, as shown in $\mathrm{Ta}$ - 
ble 1, second row.

Our calculations predict a significant reduction of the electronic heat coefficient $(\gamma)$ for UN (B3LYP result), as shown in Table 1. It would be interesting to examine an experimental evaluation although recently Parker et al., using the experimental fit [15] for ThN, also found $\gamma$ to be very small $\left(0.001 \mathrm{~J} \cdot \mathrm{mol}^{-1} \cdot \mathrm{K}^{-2}\right)$.

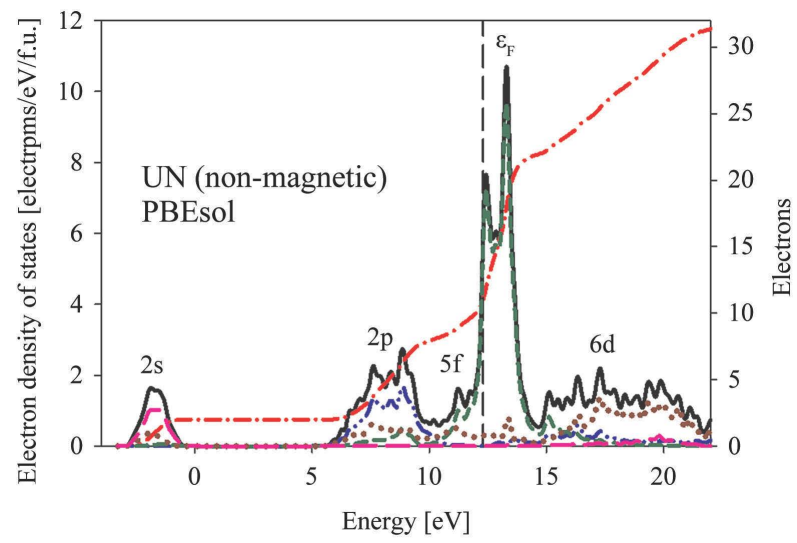

(a)

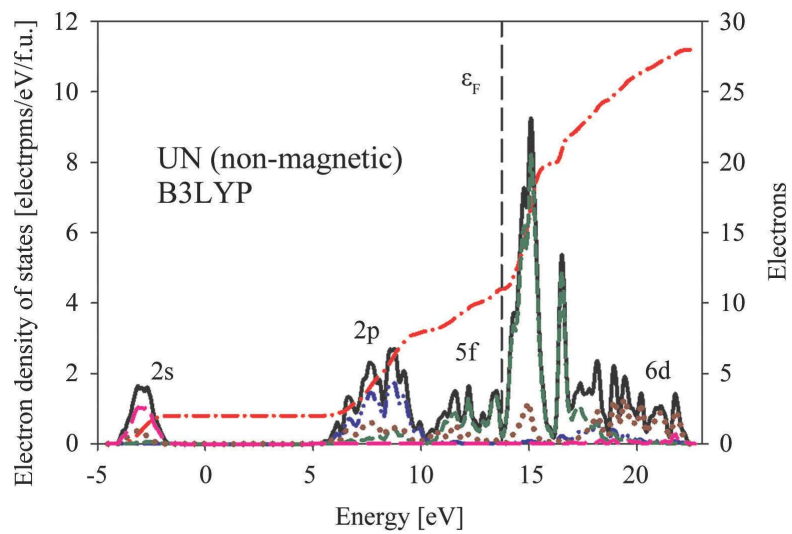

(b)

Figure 1. The calculated total (black solid line) electron densities of states of UN using (a) PBEsol functional and (b) non-local hybrid functional (B3LYP) are presented. The projected partial electron densities of states of nitrogen $2 p$ (dashed-dot-dot blue line), and 2s (dashed-long, pink line) and for uranium: 6d (dark red dotted line) and 5f (dashed medium green line) electron densities of states are shown as indicated. The dashed grey lines indicate the Fermi energy. The integrated total number of electrons (left y axis) as a function of energy is indicated as dashed-dot red lines.

Table 1. The comparison of the calculated electron density of states at Fermi energy, the electronic heat capacity coefficient, and Fermi Energy of UN using PBEsol and B3LYP functionals.

\begin{tabular}{ccc}
\hline Calc. QE & $\mathrm{UN}$ & $\mathrm{UN}$ \\
This work & $($ PBEsol $)$ & $(\mathrm{B} 3 \mathrm{LYP})$ \\
\hline$\rho\left(\boldsymbol{\varepsilon}_{F}\right)[$ electr./eV/FU] & 7.472 & 0.253 \\
$\gamma\left[\mathrm{J} \cdot \mathrm{mol}^{-1} \cdot \mathrm{K}^{-2}\right]$ & 0.0176 & 0.0006 \\
$E_{F}[\mathrm{eV}]$ & 12.31 & 13.72 \\
\hline
\end{tabular}




\subsection{Electronic Resistivity}

We calculated phonons' dispersion and the density of states as before [5] but used a finer grid: $6 \times 6 \times 6$. Next, using EPW code, we calculated the Eliashberg transport coupling function: $\alpha_{t r}^{2} F(\omega)$ (Equation 55 in Ref. [10]) together with the integrated electron-phonon strength $(\lambda)$ as a function of frequency $(\omega)$ using Equation (3). In Figure 2, we present the results by a dashed red line and dashed-dot blue line, respectively. The integration was done on a $48 \times 48 \times 48$ homogeneous k-point mesh and a $48 \times 48 \times 48$ homogeneous q-point mesh with Gaussian smearing of $100 \mathrm{meV}$ for the electrons, and $0.1 \mathrm{meV}$ for the phonons. The total integrated electron-phonon strength of 0.2472 only slightly increases $(0.2516)$ when $36 \times 36 \times 36$ grids are used while the Eliashberg transport coupling function (indicated by a solid black line), which is presented here using 0.5 $\mathrm{meV}$ smearing, shows a more visible effect of grid change (Figure 2).

Next, we calculated the electrical resistivity of $\mathrm{UN}$ and the number of mobility electrons using Equations (2) and (4), and the results are presented in Figure 3 and Figure 4. We used porosity-free experimental resistivity for UN by Hayes et al. [16]. We note that both the resistivity and the evaluated number of mobility electrons are not much affected by a change in the grid. The averages over a number of mobility electrons in the temperature range $300 \mathrm{~K}-1000 \mathrm{~K}$ are slightly higher for the finer grid ( $n_{a r}: 0.343$ e versus $0.337 \mathrm{e}$ ). We found that $\mathrm{Zi}$ man's formula (Equation (2)) predicts a stronger decrease of resistivity for decreasing temperatures than experiment when assuming that the number of mobility electrons is constant and equal to $n_{a b}$ as indicated in Figure 3. To reproduce the experimentally observed temperature dependence of the resistivity of UN as presented in Figure 3 (black solid line), a variable with a temperature-dependent number of electrons was needed, as evaluated in Figure 4.

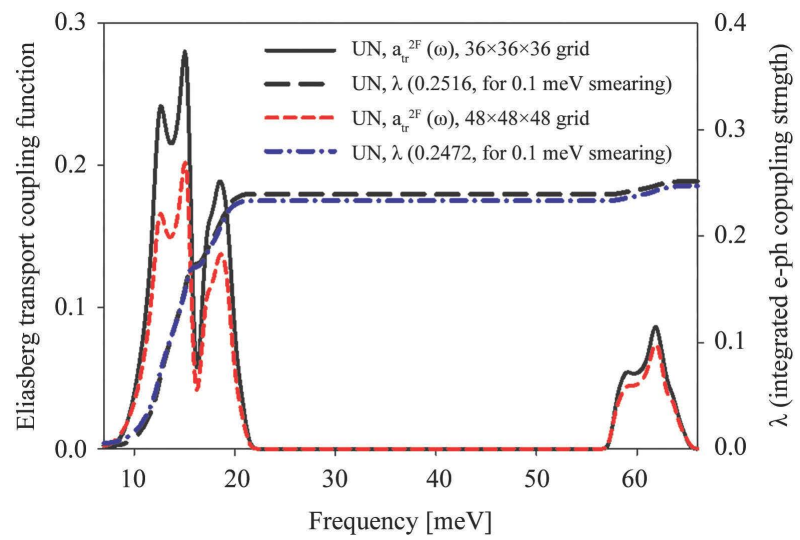

Figure 2. The calculated Eliashberg transport coupling function for UN: $\alpha_{t r}^{2} F(\omega)$ together with the integrated electron-phonon strength $(\lambda)$ as a function of frequency $(\omega)$ using the same homogeneous grid for k-point and q-point meshes: 48 $\times 48 \times 48$ is shown by dashed red line while for the $36 \times 36 \times 36$ grid a solid black line is used. The respective integrated electron-phonon strengths (right y axis) are indicated by the dashed-dot blue line and dashed long black line, respectively. 


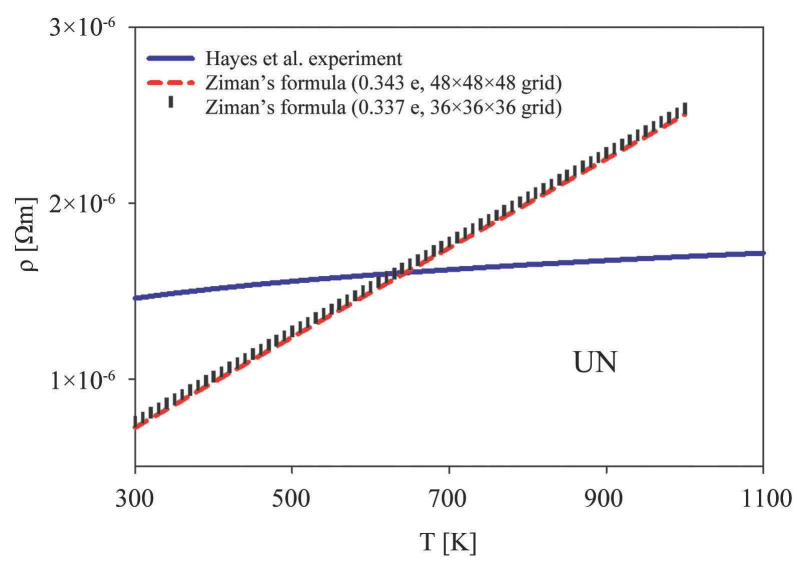

Figure 3. The calculated electrical resistivity of UN using Equation (2) for two grids as indicated and a constant number of mobility electrons $\left(n_{\text {av }}\right): 0.343$ e and 0.337 , respectively, versus experimental correlations [16] are shown, as indicated.

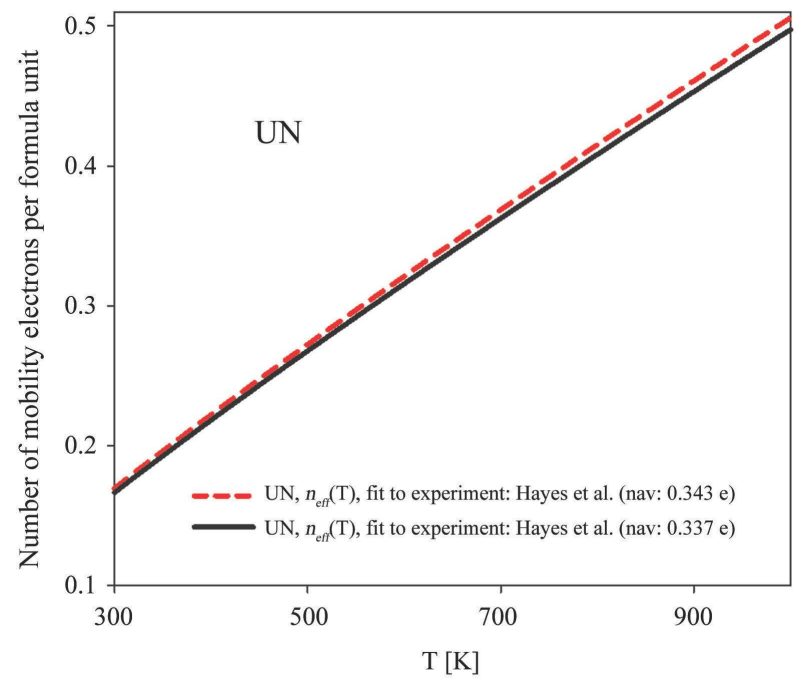

Figure 4. The evaluated temperature dependence of the number of mobility electrons of UN as a function of temperature, which reproduces the experimental resistivity [16] for Ziman's formula (Equation (2)).

\subsection{Thermal Conductivity}

In Figure 5, the electronic thermal conductivity of UN, as calculated from the experimental resistivity of UN [16], via Wiedemann-Franz law [13] Equation (5), is shown by a solid black line.

We also calculated the electronic thermal conductivity of UN, using Equation (5) and the calculated resistivity shown in Figure 3 for the constant number of mobility electrons $\left(n_{a v}\right): 0.343 \mathrm{e}$ and $0.337 \mathrm{e}$, respectively. They are indicated by the dashed red line and vertical, black marks, respectively.

It can be noted that the derived thermal conductivity for the assumed constant number of mobility electrons is almost independent of temperature and behaves similarly to that studied by us for Al [6]. Therefore, to reproduce the experimentally observed strong temperature dependence (indicated by black, solid line) of 


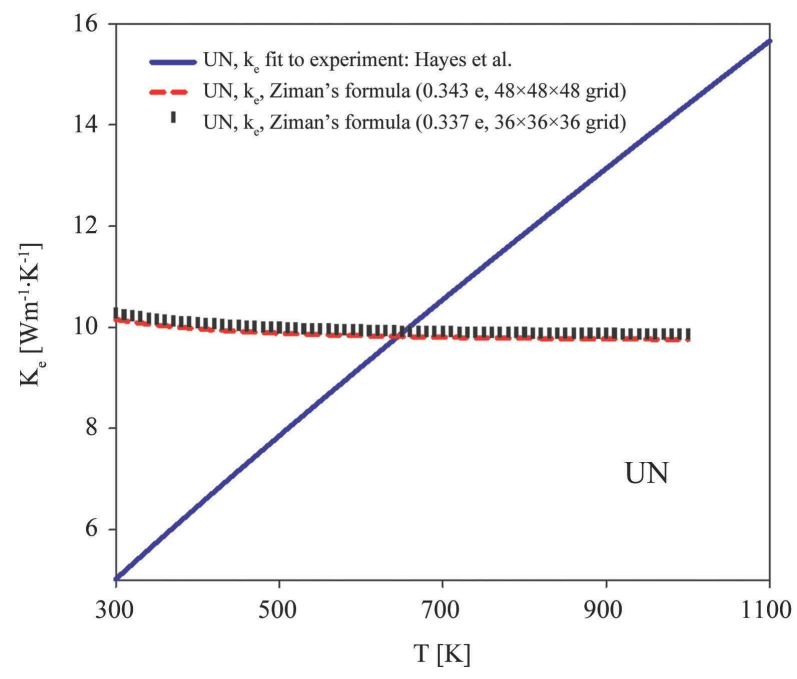

Figure 5. The calculated electronic thermal conductivities of UN, using Equation (5) and the calculated (presented above in Figure 3 ) resistivity for the constant number of mobility electrons $\left(n_{a v}\right): 0.343$ e and 0.337 e are indicated by dashed red line and vertical, black marks, respectively. The black solid line represents the electronic thermal conductivity calculated from the experimental resistivity [16] and Equation (5) or alternatively using Equation (2) with the derived number of mobility electrons shown in Figure 4.

the electronic thermal conductivity of UN it is necessary to use the number of mobility electrons that increase with the increasing temperature, as presented in Figure 4. Further experimental investigation of the number of mobility electrons in $\mathrm{UN}$ as a function of temperature is of interest.

\section{Summary}

We have investigated the electronic heat capacity, thermal conductivity, and resistivity of UN using Quantum Espresso and EPW code. GGA, PBEsol functional, and non-local hybrid functional (B3LYP) were implemented. The calculated electronic heat coefficient was found to be significantly reduced $\left(0.0176 \mathrm{~J} \cdot \mathrm{mol}^{-1} \cdot \mathrm{K}^{-2}\right.$ versus $0.0006 \mathrm{~J} \cdot \mathrm{mol}^{-1} \cdot \mathrm{K}^{-2}$ ) when the non-local hybrid functional (B3LYP) was used.

Furthermore, we found that the calculated electrical resistivity using Ziman's formula for metals with the Eliashberg transport coupling function as implemented in EPW code for non-spin-polarized calculations, would only reproduce the experimental results for UN when the derived number of mobility electrons, which increases with increasing temperature, was used. This also applies to the evaluated electronic thermal conductivity, which for any assumed constant number of carriers in UN would be not increasing with the increasing temperature but would remain almost independent of temperature, like $\mathrm{Al}$ for example.

\section{Acknowledgements}

The authors acknowledge access to high-performance supercomputers at Compute Canada (Calcul Quebec, West Grid and SHARCNET).

Free access to Quantum Espresso, EPW, with technical support is acknowl- 
edged. The first author acknowledges a very constructive discussion with Dr. Samuel Poncé.

This work was supported by the Discovery grant from the National Sciences and Engineering Research Council of Canada.

\section{Conflicts of Interest}

The authors declare no conflicts of interest regarding the publication of this paper.

\section{References}

[1] Pioro, I.L., Khan, M., Hopps, V., et al. (2008) Journal of Power and Energy Systems, 2, 874-888. https://doi.org/10.1299/jpes.2.874

[2] OECD (2018) Nuclear Science 2018, NEA No. 7317, @, State-of-the-Art Report on Light Water Reactor Accident-Tolerant Fuels. 367.

[3] Brown, N.R., Gorton, J.P., Collins, B.S. and Nelson, A.T. (2019) Nuclear Engineering and Design, 355, Article ID: 110317. https://doi.org/10.1016/j.nucengdes.2019.110317

[4] Szpunar, B. and Szpunar, J.A. (2014) International Journal of Nuclear Energy, 2014, Article ID: 178360. https://doi.org/10.1155/2014/178360

[5] Szpunar, B., Ranasinghe, J.I., Malakkal, L. and Szpunar, J.A. (2020) Journal of Physics and Chemistry of Solids, 146, Article ID: 109636.

https://doi.org/10.1016/j.jpcs.2020.109636

[6] Ranasinghe, J.I., Szpunar, B., Jossou, E., Malakkal, L. and Szpunar, J.A. (2018) The ASME Journal of Nuclear Engineering and Radiation Science, 4, Article ID: 031020. https://doi.org/10.1115/1.4039886

[7] Szpunar, B. and Szpunar, J.A. (2013) Journal of Nuclear Materials, 439, 243-250. https://doi.org/10.1016/j.jnucmat.2012.10.009

[8] Becke, A.D. (1993) The Journal of Chemical Physics, 98, 5648-5652. https://doi.org/10.1063/1.464913

[9] Giannozzi, P., et al. (2009) Journal of Physics. Condensed Matter, 21, $395502-$ 395521. https://doi.org/10.1088/0953-8984/21/39/395502

[10] Poncé, S., Margine, E.R., Verdia, C. and Giustinoa, F. (2016) Computer Physics Communications, 209, 116-133. https://doi.org/10.1016/j.cpc.2016.07.028

[11] Perdew, J.P., Ruzsinszky, A., Csonka, G.I., Vydrov, O.A., Scuseria, G.E., Constantin, L.A., Zhou, X. and Burke, K. (2008) Physical Review Letters, 100, Article ID: 136406. (Erratum: Physical Review Letters, 102 (2009) 039902 p. 1)

[12] Baroni, S., Giannozzi, P. and Testa, A. (1987) Physical Review Letters, 58, 1861 1864. https://doi.org/10.1103/PhysRevLett.58.1861

[13] Wiedemann, F.R. (1853) Annalen der Physik, 165, 497-531. (In German) https://doi.org/10.1002/andp.18531650802

[14] Knott, H.W., Lander, G.H., Mueller, M.H. and Vogt, O. (1980) Physical Review B, 21, 4159-4165. https://doi.org/10.1103/PhysRevB.21.4159

[15] Parker, S.S., White, J.T., Hosemann, P. and Nelson, A.T. (2019) Journal of Nuclear Materials, 526, Article ID: 151760. https://doi.org/10.1016/j.jnucmat.2019.151760

[16] Hayes, S.L., Thomas, J.K. and Peddicord, K.L. (1990) Journal of Nuclear Materials, 171, 289-299. https://doi.org/10.1016/0022-3115(90)90376-X 